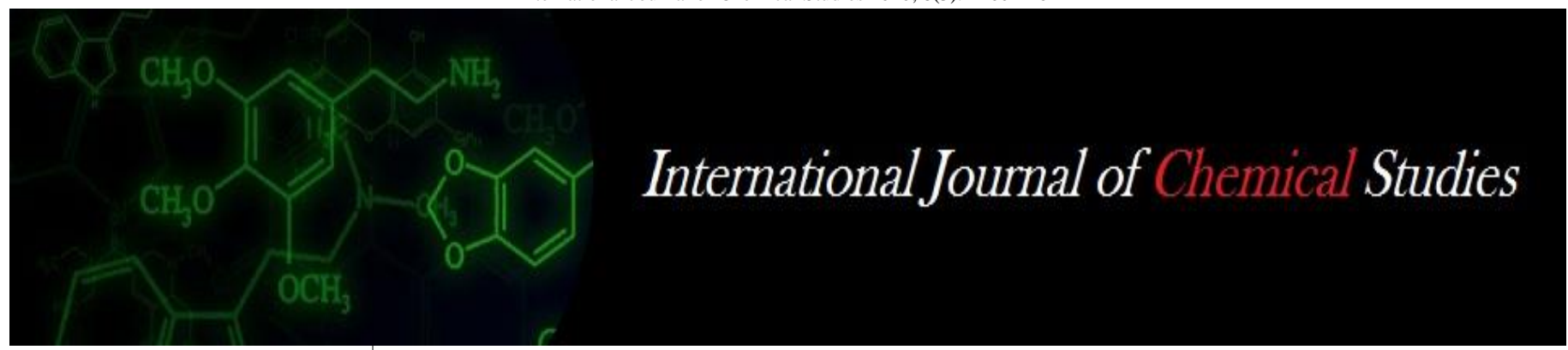

P-ISSN: 2349-8528

E-ISSN: 2321-4902

www.chemijournal.com

IJCS 2020; 8(3): 2260-2264

(C) 2020 IJCS

Received: 28-03-2020

Accepted: 30-04-2020

Sonali Gaur

Assistant Professor School of

Agriculture Uttaranchal

University, Dehradun,

Uttarakhand, India

Rajneesh Bhardwaj

Assistant Professor, School of

Agriculture Graphic Era Hill

University, Dehradun,

Uttarakhand, India

\section{Anju Arora}

JRO, Department of Genetics and Plant Breeding, GBPUA\&T

Pantnagar G.B. Pant University of Agriculture and Technology,

Pantnagar, Uttarakhand, India

\section{AK Gaur}

Research Scholar, Department of Genetics and Plant Breeding

GBPUA\&T Pantnagar

G.B. Pant University of

Agriculture and Technology,

Pantnagar, Uttarakhand, India

\section{Estimation of combining ability and heterosis by using diallel mating design in Kabuli genotypes of chickpea}

\author{
Sonali Gaur, Rajneesh Bhardwaj, Anju Arora and AK Gaur
}

DOI: https://doi.org/10.22271/chemi.2020.v8.i3af.9548

\begin{abstract}
An experiment consisting of six parents and 15 hybrids (developed by using 6 x 6 half diallel mating design) in kabuli chickpea was laid out in RBD design with three replications to estimate combining ability and heterosis. The ANOVA (Analysis of Variance) for different characters revealed highly significant differences among genotypes for all traits under study.The estimates of SCA variance were found to be higher than the GCA variance for all the characters under consideration, indicating good prospects for exploitation of non-additive gene action for grain yield and its components under study. Estimates of the ratio of $g c a$ variances to the $s c a$ variances for all the characters is less than one indicating the preponderance of non-additive gene effects (dominance and epistasis) in controlling the expression of all the characters under study. Line KAK2 was identified as good general combiner for plant height, canopy width, number of secondary branches per plant, number of pods per plant, pod length, grain yield per plant, biological yield per plant, 100 seed weight and harvest index. High estimates of heterosis over better parent, mid parent and standard check was exhibited by three crosses viz., PKC1 × HK4, KAK2 × HK4 and JGK1 × HK4.
\end{abstract}

Keywords: Chickpea, kabuli, diallel, GCA, SCA and heterosis

\section{Introduction}

Chickpea (Cicer arietinum $\mathrm{L}$.) with $2 \mathrm{n}=2 \mathrm{x}=16$ stands as third most important food legume among the essential sustenance legumes grown worldwide after dry bean and dry pea. Chickpea is said to be one of the first grain legumes domesticated by humans in the old world (Van der Maesen, 1972) ${ }^{[9]}$. It belongs to the family Fabaceae and sub family Faboideae and is only cultivated species of the genus Cicer. Chickpea is classified into two distinct types: the desi type with pink flowers, anthocyanin pigmentation on stems small, angular, dark coloured seeds and the kabuli type with large, white flowers, lacking anthocyanin pigmentation on stems, smooth coated, beige seeds. This classification overlaps, to a particular extent, with the macrosperma and microsperma races proposed by (Moreno and Cubero, 1978) ${ }^{[6]}$ utilizing quantitative as well as qualitative characters. Information on genetic frameworks governing the inheritance of traits, combining ability and hybrid vigour helps not just in the identification of superior parents for breeding programmes but also helps in the identification of potential crosses for further exploitation. For a successful breeding programme, it is also a pre-requisite to evaluate nature of gene action implicated in expression of the various agronomical vital traits and determining the potentiality of the parents in hybrid combinations. In this respect, the information of combining ability is rather important in identifying the parents for their utilization in crop improvement programme. It additionally gives essential and fundamental information concerning nature of gene action that governs expression of the character being referred to and thus helps in deciding upon the future breeding strategy. Heterosis breeding in crop plants has been the best approach among different specialized choices accessible to the geneticists and plant breeders for improvement of productivity in crop plants. This phenomenon though not completely understood genetically up until now, has yet empowered the plant researchers to enhance the performance of several economic traits. Heterosis in chickpea has been perceived as a method for improving yield and other critical traits. The extent of heterosis especially for yield is of ultimate significance, and if the heterosis is of high magnitude, it can accomplish high productivity and in this manner high yield levels.
Corresponding Author: Rajneesh Bhardwaj

Assistant Professor, School of Agriculture Graphic Era Hill

University, Dehradun,

Uttarakhand, India 


\section{Material and Methods}

The present investigation was undertaken with six Kabuli chickpea genotypes (PG071, PKC1, KAK2, JGK1, GNG1969 and HK4) which were crossed in a half diallel fashion to produce fifteen $\mathrm{F}_{1}$ 'sduring rabi season 2014-15 and were evaluated in the following year during rabi season 2015-16 in a Randomized Complete Block Design with three replication. Observations were recorded for days to 50 per cent flowering, days to maturity, plant height, number of primary branches per plant, number of secondary branches per plant, number of pods per plant, Plant canopy width, Pod length, number of seeds per pod, 100 seed weight, Harvest index (\%), grain yield per plant and biological yield per plant. The combining ability analysis for parental genotypes and their crosses were carried out following method 2 and model I (fixed effect model) of Griffing (1956) [3]. Heterosis was computed for each character as suggested by Hayes et al. (1955) ${ }^{[4]}$ and Fonseca and Paterson (1968) ${ }^{[2]}$. For testing the significance of heterosis, the method proposed by Panse and Sukhatme (1961) ${ }^{[8]}$ were followed.

\section{Results and Discussion}

The analysis of variance showed highly significant differences among the parents for each of the thirteen characters, showing the presence of sufficient genetic variation in all the six parents and their F1's under the study (Table 1). The analysis of variance for combining ability indicated that mean squares due to GCA and SCA were significant for all the characters studied (Table 2). This highlighted the importance of both additive and non-additive gene action for the expression of the traits. The magnitude of the non-additive (SCA) variance was found to be higher than additive (GCA) variance for all the characters studied (Table 2). Estimates of the ratio of GCAvariances to the SCA variances for all the characters is less than one indicating the preponderance of non-additive gene effects (dominance and epistasis) in controlling the expression of all the characters under study. These results were in agreement with the

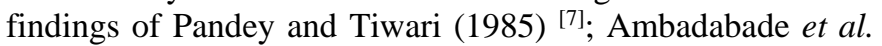
(2014); Jadhav and Gawande (2016) ${ }^{[5]}$.

The overall estimates of GCAeffects revealed (Table 3 ) that none of the parent was good general combiner for all the attributes studied. However, parental line KAK2 was identified as good general combiner for plant height, canopy width, number of secondary branches per plant, number of pods per plant, pod length, grain yield per plant, biological yield per plant, 100 seed weight and harvest index. Parental line PKC1 appeared as good general combiner for days to maturity, number of primary branches per plant, number of pods per plant and pod length; KAK2 for plant height, canopy width, number of secondary branches per plant, number of pods per plant, pod length, grain yield per plant ,biological yield per plant 100 seed weight and harvest index; JGK1for days to $50 \%$ flowering, days to maturity; GNG1969 for days to $50 \%$ flowering, number of primary branches per plant, number of secondary branches per plant, number of pods per plant and number of seeds per pod .Parent HK4 was found to be a good general combiner for pod length, grain yield per plant, biological yield per plant,100 seed weight and harvest index.

Thus among the parental lines, KAK2 can be ranked as the best parent as it had a good general combining ability for maximum number (9) of yield attributes including grain yield per plant. Parent PG071 had good GCAfor maximum number (8) of yield attributes after KAK2 followed by HK4, which showed good GCAfor 5 yield attributes

None of the crosses was found having significantSCA effects for all the traits. Significant positive or negative SCA effects were observed in $F_{1}$ generation for yield and various yield attributing traits. Cross PG071 $\times$ PKC1 was identified as the best cross for canopy width, number of pods per plant and number of seeds per pod, as per SCA effect and per se performance. The cross PKC1 $\times$ HK 4 had highest SCA effect and per se performance for days to maturity, pod length, grain yield per plant and harvest index. Cross KAK2 × GNG1969 was identified as best cross for number of secondary branches per plant based on highest SCA effect and per se performance.

\section{Heterosis}

The percentage of heterosis over mid parent (relative heterosis), better parent (heterobeltiosis) and standard check variety (standard heterosis) were estimated for all the 15 hybrids for thirteen biometrical characters and were presented in (Table 4).The results of heterosis for yield per plant indicated that, high estimates of heterosis over better parent, mid parent and standard check was exhibited by three crosses $\mathrm{PKC} 1 \times \mathrm{HK} 4, \mathrm{KAK} 2 \times \mathrm{HK} 4$ and JGK $1 \times \mathrm{HK} 4$. The cross KAK2 $\times$ HK4 also expressed significant heterosis for plant height, number of secondary branches, number of pods per plant, number of seeds per pod and 100 seed weight over all the three parents. HK4 for canopy width, number of secondary branches, pod length, grain yield per plant, 100 seed weight and harvest index.

Table 1: Analysis of variance for thirteen characters in chickpea

\begin{tabular}{|c|c|c|c|c|c|}
\hline \multirow{2}{*}{ Character } & \multicolumn{3}{|c|}{ Mean sum squares } & \multirow{2}{*}{ S.E } & \multirow{2}{*}{ CV (\%) } \\
\hline & Replication & Treatment & Error & & \\
\hline Degree of freedom & 01 & 20 & 20 & & \\
\hline Days to $50 \%$ flowering & 0.21 & $34.78 * *$ & 1.41 & 0.84 & 1.60 \\
\hline Days to maturity & 2.38 & $166.42 * *$ & 2.73 & 1.16 & 1.17 \\
\hline Plant height $(\mathrm{cm})$ & 10.78 & $125.63 * *$ & 6.15 & 1.75 & 7.99 \\
\hline Canopy width $(\mathrm{cm})$ & 0.11 & $18.39 * *$ & 0.40 & 0.44 & 3.26 \\
\hline Number of primary branches per plant & 0.02 & $0.63^{* *}$ & 0.04 & 0.15 & 7.28 \\
\hline Number of secondary branches per plant & 0.07 & $0.77 * *$ & 0.07 & 0.19 & 8.48 \\
\hline Number of pods per plant & 2.16 & $90.65 * *$ & 2.29 & 1.07 & 4.68 \\
\hline Pod length $(\mathrm{cm})$ & 0.03 & $10.58 * *$ & 0.37 & 0.43 & 2.83 \\
\hline Number of seeds per pod & 0.02 & $0.03 * *$ & 0.007 & 0.06 & 8.03 \\
\hline Grain yield per plant $(\mathrm{g})$ & 2.62 & $4.01 * *$ & 0.83 & 0.64 & 10.47 \\
\hline Biological yield per plant (g) & 6.16 & $82.77 * *$ & 13.21 & 2.57 & 12.48 \\
\hline 100 seed weight $(\mathrm{g})$ & 0.97 & $34.58 * *$ & 0.53 & 0.51 & 2.82 \\
\hline Harvest index $(\%)$ & 5.32 & $73.11 * *$ & 6.32 & 1.77 & 7.98 \\
\hline
\end{tabular}

** Significant at $1 \%$ level of probability, * significant at $5 \%$ level of probability 
Table 2: Analysis of variance for combining ability for different characters

\begin{tabular}{|c|c|c|c|c|c|c|}
\hline \multirow{2}{*}{ Character } & \multicolumn{3}{|c|}{ Mean sum squares Source of variation } & \multirow{3}{*}{$\sigma^{2}$ gca } & \multirow{3}{*}{$\sigma^{2}$ sca } & \multirow{3}{*}{$\sigma^{2} \mathrm{gca} / \sigma^{2} \mathrm{sca}$} \\
\hline & GCA & SCA & Error & & & \\
\hline Degree of Freedom & 5 & 15 & 20 & & & \\
\hline Days to $50 \%$ flowering & $44.74 * *$ & $31.46 * *$ & 1.41 & 1.66 & 30.05 & 0.06 \\
\hline Days to maturity & $218.24 * *$ & $149.14 * *$ & 2.73 & 8.64 & 146.41 & 0.06 \\
\hline Plant height $(\mathrm{cm})$ & $150.90 * *$ & $117.20 * *$ & 6.15 & 4.21 & 111.05 & 0.04 \\
\hline Canopy width $(\mathrm{cm})$ & $29.25 * *$ & $14.77 * *$ & 0.40 & 1.81 & 14.37 & 0.13 \\
\hline Number of primary branches per plant & $1.01 * *$ & $0.86 * *$ & 0.66 & 0.01 & 0.81 & 0.01 \\
\hline Number of secondary branches per plant & $2.03 * *$ & $1.65 * *$ & 0.07 & 0.04 & 1.57 & 0.03 \\
\hline Number of pods per plant & $131.20 * *$ & $77.12 * *$ & 2.29 & 6.76 & 74.83 & 0.09 \\
\hline Pod length $(\mathrm{cm})$ & $12.32 * *$ & $10.00 * *$ & 0.37 & 0.29 & 9.64 & 0.03 \\
\hline Number of seeds per pod & $0.04 * *$ & $0.03 * *$ & 0.007 & 0.0006 & 0.03 & 0.02 \\
\hline Grain yield per plant $(\mathrm{g})$ & $4.824 * *$ & $3.74 * *$ & 0.83 & 0.13 & 2.92 & 0.05 \\
\hline Biological yield per plant $(\mathrm{g})$ & $95.10 * *$ & $78.66 * *$ & 13.21 & 2.05 & 65.45 & 0.03 \\
\hline 100 seed weight $(\mathrm{g})$ & $38.04 * *$ & $33.43 * *$ & 0.53 & 0.58 & 32.90 & 0.02 \\
\hline Harvest index $(\%)$ & $87.99 * *$ & $68.15 * *$ & 6.32 & 2.48 & 61.83 & 0.04 \\
\hline
\end{tabular}

** Significant at $1 \%$ level of probability * significant at $5 \%$ level of probability $\delta^{2}{ }_{\text {gca }}=$ variance of GCA $\delta^{2}$ sca $=$ Variance of SCA

Table 3: Summary table for general combining ability of parents of chickpea

\begin{tabular}{|c|c|c|c|c|c|c|c|c|c|c|c|c|c|c|}
\hline \multirow[b]{2}{*}{ Parents } & \multicolumn{13}{|c|}{ Characters } & \multirow[b]{2}{*}{$\begin{array}{c}\text { Overall } \\
\text { good }\end{array}$} \\
\hline & $\begin{array}{c}\text { Days to } 50 \\
\% \\
\text { flowering }\end{array}$ & $\begin{array}{c}\text { Days to } \\
\text { maturity }\end{array}$ & $\begin{array}{c}\text { Plant } \\
\text { height } \\
(\mathrm{cm})\end{array}$ & $\begin{array}{c}\text { Canopy } \\
\text { width } \\
(\mathrm{cm})\end{array}$ & $\begin{array}{c}\text { Number of } \\
\text { primary } \\
\text { branches } \\
\text { per plant }\end{array}$ & \begin{tabular}{|c|} 
Number of \\
secondary \\
branches per \\
plant
\end{tabular} & $\begin{array}{c}\text { Number } \\
\text { of pods } \\
\text { per plant }\end{array}$ & $\begin{array}{c}\text { Pod } \\
\text { length } \\
(\mathrm{cm})\end{array}$ & $\begin{array}{l}\text { Number } \\
\text { of seeds } \\
\text { per pod }\end{array}$ & \begin{tabular}{|} 
Grain \\
yield per \\
plant $(g)$
\end{tabular} & $\begin{array}{l}\text { Biological } \\
\text { yield per } \\
\text { plant }(g)\end{array}$ & $\begin{array}{c}100 \text { seed } \\
\text { weight }(g)\end{array}$ & $\begin{array}{c}\text { Harvest } \\
\text { index } \\
(\%)\end{array}$ & \\
\hline PG071 & $\mathrm{A}$ & $\mathrm{P}$ & $\mathrm{G}$ & $\mathrm{G}$ & $\mathrm{G}$ & $\mathrm{A}$ & $\mathrm{G}$ & $\mathrm{G}$ & $\mathrm{A}$ & $\mathrm{G}$ & $\mathrm{G}$ & $\mathrm{P}$ & $\mathrm{G}$ & 08 \\
\hline $\mathrm{PKC1}$ & $\mathrm{P}$ & $\mathrm{G}$ & $\mathrm{A}$ & $\mathrm{P}$ & $\mathrm{G}$ & $\mathrm{P}$ & $\mathrm{G}$ & $\mathrm{G}$ & $\mathrm{A}$ & $\mathrm{P}$ & $\mathrm{A}$ & $\mathrm{P}$ & $\mathrm{A}$ & 04 \\
\hline KAK2 & $\mathrm{A}$ & $\mathrm{P}$ & $\mathrm{G}$ & $\mathrm{G}$ & $\mathrm{A}$ & $\mathrm{G}$ & $\mathrm{G}$ & $\mathrm{G}$ & $\mathrm{A}$ & $\mathrm{G}$ & $\mathrm{G}$ & $\mathrm{G}$ & $\mathrm{G}$ & 09 \\
\hline JGK1 & $\mathrm{G}$ & $\mathrm{G}$ & $\mathrm{P}$ & $\mathrm{P}$ & $\mathrm{P}$ & A & $\mathrm{P}$ & $\mathrm{P}$ & $\mathrm{P}$ & $\mathrm{P}$ & $\mathrm{P}$ & $\mathrm{P}$ & $\mathrm{P}$ & 02 \\
\hline \begin{tabular}{|l|} 
GNG1969 \\
\end{tabular} & $\mathrm{G}$ & $\mathrm{P}$ & $\mathrm{A}$ & $\mathrm{P}$ & $\mathrm{G}$ & $\mathrm{G}$ & $\mathrm{G}$ & $\mathrm{A}$ & $\mathrm{G}$ & $\mathrm{A}$ & $\mathrm{A}$ & $\mathrm{P}$ & $\mathrm{P}$ & 05 \\
\hline HK4 & $\mathrm{P}$ & $\mathrm{P}$ & $\mathrm{A}$ & $\mathrm{P}$ & $\mathrm{A}$ & $\mathrm{P}$ & $\mathrm{P}$ & $\mathrm{G}$ & $\mathrm{A}$ & $\mathrm{G}$ & $\mathrm{G}$ & $\mathrm{G}$ & $\mathrm{G}$ & 05 \\
\hline
\end{tabular}

Table 4: Percentage increase or decrease of the $F_{1}$ over mid parent (relative heterosis), better parent (heterobeltiosis) and standard parent (economic heterosis)

\begin{tabular}{|c|c|c|c|c|c|c|c|c|c|c|c|c|}
\hline \multirow{3}{*}{ Crosses } & \multirow{2}{*}{\multicolumn{3}{|c|}{$\begin{array}{c}\text { Days to 50\% flowering } \\
\text { Heterosis over }\end{array}$}} & \multirow{2}{*}{\multicolumn{3}{|c|}{\begin{tabular}{|c|} 
Days to maturity \\
Heterosis over \\
\end{tabular}}} & \multirow{2}{*}{\multicolumn{3}{|c|}{\begin{tabular}{c|} 
Plant height $(\mathbf{c m})$ \\
Heterosis over \\
\end{tabular}}} & \multirow{2}{*}{\multicolumn{3}{|c|}{$\begin{array}{c}\text { Canopy width }(\mathrm{cm}) \\
\text { Heterosis over }\end{array}$}} \\
\hline & & & & & & & & & & & & \\
\hline & $\begin{array}{c}\text { Mid } \\
\text { parent }\end{array}$ & $\begin{array}{l}\text { Better } \\
\text { parent }\end{array}$ & $\begin{array}{c}\text { Standard } \\
\text { parent }\end{array}$ & $\begin{array}{c}\text { Mid } \\
\text { parent }\end{array}$ & $\begin{array}{l}\text { Better } \\
\text { parent }\end{array}$ & $\begin{array}{c}\text { Standard } \\
\text { parent }\end{array}$ & $\begin{array}{c}\text { Mid } \\
\text { parent }\end{array}$ & $\begin{array}{l}\text { Better } \\
\text { parent }\end{array}$ & $\begin{array}{r}\text { Stand } \\
\text { pare }\end{array}$ & $\begin{array}{c}\text { Mid } \\
\text { parent }\end{array}$ & $\begin{array}{l}\text { Better } \\
\text { parent }\end{array}$ & Stad \\
\hline $\bar{P}$ & .97 & $-2.51 *$ & ** & $8.34 * *$ & & $12.74 * *$ & $16.28 * *$ & $12.20 * *$ & $19.32 * *$ & $25.06 * *$ & $8.35 * *$ & (5) \\
\hline PG07 & $92 * *$ & $-10.06 * *$ & & $4.91 * *$ & & & $-8.54 *$ & $27 * *$ & & $-12.28 * *$ & $-14.62 * *$ & $-14.62 * *$ \\
\hline PG0 & $79 * *$ & $6.20 * *$ & & $13.19 * *$ & 2 & & $-49.23 * *$ & $2 * *$ & $-55.57 * *$ & $35.44 * *$ & $7.30 * *$ & \\
\hline$\overline{\mathrm{PG} 0}$ & -1.92 & $-13.33 * *$ & -13 & $-4.56 *$ & & & $-12.89 * *$ & & 14 & $-8.36 * *$ & $-16.49 * *$ & \\
\hline & 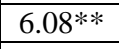 & $3.97 * *$ & & $27 * *$ & & & $15.30^{* * *}$ & & & $-10.95 * *$ & $-21.08 * *$ & \\
\hline & $.57 * *$ & $-12.57 * *$ & & $20.07 * *$ & & $17.76 * *$ & $-33.47 * *$ & $5 * *$ & & $-27.18 * *$ & $-38.33 * *$ & \\
\hline & $.21 * *$ & $-7.58 * *$ & $2 * *$ & $8.71 * *$ & & & $-29.46^{* *}$ & -40 & -40 & $13.15 * *$ & $1.70^{*}$ & -2 \\
\hline $\mathrm{PKC}$ & $08 * *$ & $-2.51 *$ & & $9.96 * *$ & 1.70 & $5.05 * *$ & $-13.82 * *$ & $-18.28 * *$ & $-9.87 * *$ & $7.65 * *$ & $1.77 *$ & $-20.75 * *$ \\
\hline & $16^{* *}$ & $-7.54 * *$ & & $-7.89 * *$ & -14.8 & -3.6 & 12.76 ** & & 2. & $-9.57 * *$ & $-11.89^{* *}$ & $-35.57 * *$ \\
\hline & $42 * *$ & $-11.32 * *$ & & $20.32 * *$ & 14. & & $28.09 * *$ & & & $-3.81 * *$ & $-25.29 * *$ & -2 \\
\hline 10 & -0.33 & $-7.54 * *$ & -7.2 & $7.24 * *$ & 1 & $* *$ & $-36.01 * *$ & $-39.00 * *$ & $2 * *$ & $-32.66 * *$ & $-40.11 * *$ & $1 * *$ \\
\hline KAK $2 x$ & 1.29 & -1.25 & -1.25 & $5.43 * *$ & -0.68 & $12.35 * *$ & $26.51 * *$ & $16.07 * *$ & $16.07 * *$ & $-16.21 * *$ & $-27.47 * *$ & $-27.47 * *$ \\
\hline JGK-1 & $7.80 * *$ & $6.61 * *$ & $-8.80 * *$ & $4.56^{*}$ & $-6.14 * *$ & $* *$ & $-8.30 *$ & $-25.60 * *$ & $-17.94 * *$ & $18.10 * *$ & 1.01 & $-21.34 * *$ \\
\hline & $7.74 * *$ & 1.32 & & $6.46 * *$ & & & $-37.34 * *$ & -42 & -52 & $22.46^{* *}$ & $7.56^{* *}$ & -21 \\
\hline GNG1969 x HK4 & $7.31 * *$ & 1.98 & $-3.14 *$ & -3.07 & -3.07 & $9.65 * *$ & $-26.20 * *$ & $-35.16 * *$ & $-28.49 *$ & 0.52 & $-2.53 * *$ & $-24.11 *$ \\
\hline
\end{tabular}

**Significant at $1 \%$ level of probability *significant at $5 \%$ level of probability

\begin{tabular}{|c|c|c|c|c|c|c|c|c|c|c|c|c|}
\hline \multirow{3}{*}{ Crosses } & \multirow{2}{*}{\multicolumn{3}{|c|}{\begin{tabular}{|c|}
$\begin{array}{c}\text { Number of primary branches } \\
\text { per plant }\end{array}$ \\
Heterosis over \\
\end{tabular}}} & \multirow{2}{*}{\multicolumn{3}{|c|}{$\begin{array}{c}\begin{array}{c}\text { Number of secondary } \\
\text { branches per plant }\end{array} \\
\text { Heterosis over } \\
\end{array}$}} & \multirow{2}{*}{\multicolumn{3}{|c|}{\begin{tabular}{|c|} 
Number of pods per plant \\
Heterosis over \\
\end{tabular}}} & \multirow{2}{*}{\multicolumn{3}{|c|}{$\begin{array}{c}\text { Pod length (cm) } \\
\text { Heterosis over }\end{array}$}} \\
\hline & & & & & & & & & & & & \\
\hline & \begin{tabular}{|c|}
$\begin{array}{c}\text { Mid } \\
\text { parent }\end{array}$ \\
\end{tabular} & & & Mid & & & Mid & & & $\begin{array}{c}\text { Mid } \\
\text { parent }\end{array}$ & & \\
\hline & & & & & & & & & & & & \\
\hline $\mathrm{PG}$ & & & & & & & & & & & & \\
\hline & & & & & & & & & & & & \\
\hline PG07 & & & & & & & & & & & & \\
\hline & & & & & & & & & & -20 & & \\
\hline & & & & & 0.12 & & & & & & & \\
\hline & & & & & S & $5 * *$ & $12.22 * *$ & $9.06 * *$ & $-8.23 * *$ & $-4.02 * *$ & $-13.72 * *$ & $-13.72 * *$ \\
\hline $\mathrm{KC1}$ & & & & $-32.83 * *$ & -38.35 & $-39.18 * *$ & & & $-11.78 * *$ & $10.90 * *$ & & $-2.49 * *$ \\
\hline PKC1 x HK4 & $3.50 * *$ & $1.72 * *$ & $-18.05 * *$ & $-27.73 * *$ & $-29.50 * *$ & $-41.89 * *$ & $18.69 * *$ & $17.35 * *$ & $-6.81 * *$ & $15.17 * *$ & $9.23 * *$ & $3.74 * *$ \\
\hline
\end{tabular}




\begin{tabular}{|c|c|c|c|c|c|c|c|c|c|c|c|c|}
\hline KAK2 x JGK-1 & $-39.68^{* *}$ & $-47.22^{* *}$ & $-47.22^{* *}$ & $-12.00^{* *}$ & $-25.67 * *$ & $-25.67 * *$ & $-28.25^{* *}$ & $-33.94^{* *}$ & $-33.94^{* *}$ & $-7.98^{* *}$ & $-10.46^{* *}$ & $-14.96^{* *}$ \\
\hline KAK2x GNG1969 & $7.46^{* *}$ & 0 & 0 & $55.10^{* *}$ & $54.05^{* *}$ & $54.05^{* *}$ & $15.84^{* *}$ & $10.36^{* *}$ & $10.36^{* *}$ & $10.57^{* *}$ & $2.12^{* *}$ & 0 \\
\hline KAK2 x HK4 & $4.68^{* *}$ & $-6.94^{* *}$ & $-6.94^{* *}$ & $31.81^{* *}$ & $17.56^{* *}$ & $17.56^{* *}$ & $41.07^{* *}$ & $25.28^{* *}$ & $25.28^{* *}$ & $-20.51^{* *}$ & $-26.40^{* *}$ & $-26.40^{* *}$ \\
\hline JGK-1x GNG1969 & $-12.06^{* *}$ & $-17.74 * *$ & $-29.16^{* *}$ & $17.74^{* *}$ & 0 & $-1.35^{* *}$ & $-28.44^{* *}$ & $-30.98^{* *}$ & $-37.5^{* *}$ & $-8.65^{* *}$ & $-10.78^{* *}$ & $-24.32^{* *}$ \\
\hline JGK-1 x HK4 & $-10.90^{* *}$ & $-9.25^{* *}$ & $-31.94^{* *}$ & $-13.76^{* *}$ & $-18.96^{* *}$ & $-36.48^{* *}$ & $4.68^{*}$ & 0.62 & $-15.34^{* *}$ & $10.30^{* *}$ & $10.08^{* *}$ & $-6.23^{* *}$ \\
\hline GNG1969 x HK4 & $27.11^{* *}$ & $20.96^{* *}$ & $4.16^{* *}$ & $25.19^{* *}$ & $12.32^{* *}$ & $10.81^{* *}$ & $26.02^{* *}$ & $17.01^{* *}$ & $5.96^{* *}$ & $7.92^{* *}$ & $5.19^{* *}$ & $-10.39^{* *}$ \\
\hline
\end{tabular}

\begin{tabular}{|c|c|c|c|c|c|c|c|c|c|c|c|c|c|c|c|}
\hline \multirow{3}{*}{ Crosses } & \multirow{2}{*}{\multicolumn{3}{|c|}{\begin{tabular}{|c} 
Number of seeds per pod \\
Heterosis over
\end{tabular}}} & \multirow{2}{*}{\multicolumn{3}{|c|}{\begin{tabular}{|c|} 
Grain yield per plant $(\mathrm{g})$ \\
Heterosis over
\end{tabular}}} & \multirow{2}{*}{\multicolumn{3}{|c|}{\begin{tabular}{|c}
$\begin{array}{c}\text { Biological yield per plant } \\
\text { (g) }\end{array}$ \\
Heterosis over \\
\end{tabular}}} & \multirow{2}{*}{\multicolumn{3}{|c|}{$\begin{array}{c}100 \text { seed weight }(\mathrm{g}) \\
\text { Heterosis over }\end{array}$}} & \multirow{2}{*}{\multicolumn{3}{|c|}{$\begin{array}{c}\text { Harvest index (\%) } \\
\text { Heterosis over }\end{array}$}} \\
\hline & & & & & & & & & & & & & & & \\
\hline & \begin{tabular}{|c|} 
Mid \\
parent
\end{tabular} & \begin{tabular}{c|} 
Better \\
parent
\end{tabular} & $\begin{array}{c}\text { Standard } \\
\text { parent }\end{array}$ & \begin{tabular}{|c|} 
Mid \\
parent
\end{tabular} & $\begin{array}{l}\text { Better } \\
\text { parent }\end{array}$ & $\begin{array}{c}\text { Standard } \\
\text { parent }\end{array}$ & \begin{tabular}{|c|} 
Mid \\
parent
\end{tabular} & $\begin{array}{l}\text { Better } \\
\text { parent }\end{array}$ & $\begin{array}{c}\text { Standard } \\
\text { parent }\end{array}$ & $\begin{array}{c}\text { Mid } \\
\text { parent }\end{array}$ & $\begin{array}{c}\text { Better } \\
\text { parent }\end{array}$ & $\begin{array}{c}\text { Standard } \\
\text { parent }\end{array}$ & \begin{tabular}{|c|} 
Mid \\
parent
\end{tabular} & \begin{tabular}{|c|} 
Better \\
parent
\end{tabular} & $\begin{array}{c}\text { Standard } \\
\text { parent }\end{array}$ \\
\hline PG071 x PKC & $33.33^{* * *}$ & $27.27 * *$ & $27.27 * *$ & $6.49 * *$ & $-5.20 * *$ & $-24.42 * *$ & 6.45 & $-12.75 * *$ & $-26.85 * *$ & 1.57 & $11.94 * *$ & $3 * *$ & $-14.33 * *$ & $-18.72 * *$ & $-42.50 * *$ \\
\hline PG071 & $-9.09 * *$ & $-9.09 * *$ & $-9.09 * *$ & $-11.79 * *$ & $-20.73 * *$ & $-20.73 * *$ & $\begin{array}{c}- \\
31.79 * *\end{array}$ & $-37.30 * *$ & $-37.30 * *$ & -1.16 & $.57 * *$ & $-3.57 * *$ & $-30.02 * *$ & $-40.26 * *$ & $-40.26 * *$ \\
\hline PG071 & $-9.09 * *$ & $-9.09 * *$ & $-9.09 * *$ & $16.32 * *$ & -1.15 & -21.1 & \begin{tabular}{|c|}
- \\
$16.92 * *$ \\
\end{tabular} & $-34.20 * *$ & $-44.83 * *$ & $-21.68 * *$ & 30. & $-33.76^{* *}$ & 5.89 & -4.74 & $-32.62 * *$ \\
\hline $\begin{array}{c}\text { PG071x } \\
\text { GNG1969 }\end{array}$ & $21.73^{* * *}$ & $16.66^{* * *}$ & $27.27 * *$ & $12.69 * *$ & $5.20 * *$ & $-16.12 * *$ & \begin{tabular}{c|}
- \\
$36.69 * *$ \\
\end{tabular} & $-39.13 * *$ & $-48.96 * *$ & $-40.90 * *$ & $-\overline{-}$ & $-45.94 * *$ & 3.57 & 0 & $-29.26 * *$ \\
\hline PG07 & $-4.76 * *$ & $-9.09 * *$ & ** & $-33.16 * *$ & $-39.43 * *$ & $-40.55 * *$ & \begin{tabular}{|c|}
- \\
$28.99 * *$ \\
\end{tabular} & -3 & $-37.05 * *$ & $-35.00 * *$ & $\begin{array}{c}- \\
36.18^{* * *} \\
\end{array}$ & ** & $51 * *$ & $-30.43 * *$ & $-43.47 * *$ \\
\hline PKC1 x KAK2 & $-4.76 * *$ & $-9.09 * *$ & $-9.09 * *$ & $3.40 * *$ & $-16.12 * *$ & $-16.12 * *$ & -3.79 & $-26.12 * *$ & $-26.12 * *$ & $-2.10 * *$ & $\begin{array}{c}- \\
16.88^{*} * *\end{array}$ & $-16.88 * *$ & $-16.03 * *$ & $-31.36 * *$ & $-31.36 * *$ \\
\hline PKC1 $x$. & $*$ & $* *$ & 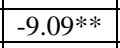 & ** & $* *$ & $* *$ & $50.23^{* *}$ & $43.76^{* * *}$ & $-22.96 * *$ & $18.05^{* *}$ & $14.69^{* * *}$ & $-15.09 * *$ & $19.60^{* * *}$ & $13.03 * *$ & $-28.22 * *$ \\
\hline PKC1x GNG1969 & $9.09 * *$ & 0 & $9.09 * *$ & $96^{* *}$ & 0.66 & $-30.41 * *$ & $\begin{array}{c}- \\
18.77 * * \\
\end{array}$ & $-31.27 * *$ & $-46.80 * *$ & 1.33 & $-9.05 * *$ & $-20.12 * *$ & $25.63 * *$ & $23.38 * *$ & $-18.75 * *$ \\
\hline PKC1 x HK4 & $10 * *$ & $10^{* * *}$ & 0 & $32.18^{* * *}$ & $7.98^{* * *}$ & $5.99 * *$ & $25.95^{* * *}$ & -0.910 & $-7.41 *$ & $23.69 * *$ & $5.59 * *$ & $4.22 * *$ & $51.85^{* *}$ & $35.25 * *$ & $9.89 * *$ \\
\hline KAK2 x JGK-1 & $-9.09 * *$ & $-9.09 * *$ & $-9.09 * *$ & $-1.77^{* *}$ & 50 ** & -2 & $-\overline{-}$ & $-35.23 * *$ & $-35.23 * *$ & -0.55 & $-13.47 *$ & -13. & $-8.29 *$ & $-28.22 * *$ & $-28.22 * *$ \\
\hline $\begin{array}{c}\text { KAK2x } \\
\text { GNG1969 }\end{array}$ & \begin{tabular}{|c|}
- \\
$13.04 * *$ \\
\end{tabular} & \begin{tabular}{|c|}
- \\
$16.66^{* * *}$ \\
\end{tabular} & $-9.09 * *$ & $12.26 * *$ & $-5.02 * *$ & $-5.06 * *$ & $21.36 * *$ & $7.65^{*}$ & $7.65^{*}$ & $-17.71 * *$ & $22.72 * *$ & $-22.72 * *$ & $-7.52 *$ & $-23.31 * *$ & $-23.31 * *$ \\
\hline KAK2 & $14.28 * *$ & $9.09 * *$ & $9.09 * *$ & $23 \cdots$ & $5.25 * *$ & $5.25^{* *}$ & $\begin{array}{c}- \\
35.17 * *\end{array}$ & $-37.30 * *$ & $-37.30 * *$ & $2.61^{* *}$ & $1.94 *$ & $1.94 *$ & $-15.27 * *$ & $-23.22 * *$ & $-23.22 * *$ \\
\hline $\begin{array}{c}\text { JGK-1x } \\
\text { GNG1969 }\end{array}$ & \begin{tabular}{|c|}
- \\
$13.04 * *$ \\
\end{tabular} & \begin{tabular}{c|}
- \\
$16.66 * *$ \\
\end{tabular} & $-9.09 * *$ & $20.29 * *$ & $8.66 * *$ & $-24.88 * *$ & $20.76 * *$ & -1.41 & $-23.69 * *$ & $15.34 * *$ & $6.28 * *$ & $-6.65^{* *}$ & -0.89 & $-7.90 * *$ & $-39.35 * *$ \\
\hline JGK-1 x HK4 & $-4.76 * *$ & $-9.09 * *$ & $-9.09 * *$ & $32.69 * *$ & $4.03 * *$ & $2.11^{* *}$ & $23.54 * *$ & $-41.74 * *$ & $-45.56^{* *}$ & $9.39 * *$ & $-4.27 * *$ & $-5.51 * *$ & $53.52 * *$ & $30.16^{* *}$ & $5.75^{* *}$ \\
\hline GNG1969 x HK4 & $18.18^{* *}$ & $8.33 * *$ & $18.18^{* *}$ & $-5.78^{* *}$ & $-19.71 * *$ & $-21.19 * *$ & $\begin{array}{c}- \\
11.52 * *\end{array}$ & -1 & $-24.42 * *$ & $* *$ & $\begin{array}{c}- \\
22.36^{* *}\end{array}$ & $-23.37 * *$ & $-14.65 * *$ & $-22.74 * *$ & $-37.23 * *$ \\
\hline
\end{tabular}

**Significant at $1 \%$ level of probability *significant at $5 \%$ level of probability

Table 5: Heterotic crosses for various characters

\begin{tabular}{|c|c|c|c|c|c|c|}
\hline S. No. & Character & \begin{tabular}{|c|} 
Promising \\
parents (per se)
\end{tabular} & \begin{tabular}{|c|} 
Good general combiners \\
(gca)
\end{tabular} & Promising cross (per se) & Good specific crosses (sca) & $\begin{array}{l}\text { Heterotic crosses (over mid, } \\
\text { better and standard parent) }\end{array}$ \\
\hline 1 & Days to $50 \%$ flowering & JGK1, GNG1969 & GNG1969, JGK-1 & $\mathrm{PKC} 1 \times \mathrm{JGK} 1$ & $\begin{array}{c}\text { PKC1 } \times \text { KAK2, PG071 } \times \\
\text { GNG1969, PKC1 } \times \text { HK4, PKC1 } \times \\
\text { JGK-1 }\end{array}$ & $\begin{array}{c}\text { PKC } 1 \times \text { JGK } 1, \text { PKC } 1 \times \text { KAK } 2 \\
\text { PKC } 1 \times \text { HK } 4\end{array}$ \\
\hline 2 & Days to maturity & JGK 1, PKC 1 & JGK-1, PKC1 & $\mathrm{PKC} 1 \times \mathrm{HK} 4$ & $\begin{array}{c}\text { PKC1 × HK4, PG071 × } \\
\text { GNG1969, PG071 × HK4 }\end{array}$ & $\mathrm{PKC} 1 \times \mathrm{HK} 4$ \\
\hline 3 & Plant height $(\mathrm{cm})$ & $\begin{array}{l}\text { GNG1969, } \\
\text { PG071 }\end{array}$ & PG071,KAK2 & $\begin{array}{c}\text { PG071 } \times \text { PKC1, KAK2 } \times \\
\text { HK4 }\end{array}$ & $\begin{array}{l}\text { KAK } 2 \times \text { JGK1, KAK2 } \times \text { HK4, } \\
\text { PG071 } \times \text { PKC1, PG071 } \times \text { HK } 4\end{array}$ & $\begin{array}{c}\text { PG071 } \times \text { PKC1, KAK2 } \times \\
\text { JGK1, KAK2 } \times \text { HK4 }\end{array}$ \\
\hline 4 & Canopy width $(\mathrm{cm})$ & KAK 2, PG071 & PG071, KAK2 & $\mathrm{PG} 071 \times \mathrm{PKC1}$ & $\begin{array}{c}\text { PG071 } \times \text { PKC1, PG071 × JGK-1, } \\
\text { JGK-1 } \times \text { HK4, JGK-1 } \times \text { GNG1969 }\end{array}$ & $\begin{array}{c}\text { PG071 × PKC1, PG071 } \times \\
\text { JGK1 }\end{array}$ \\
\hline 5 & $\begin{array}{l}\text { Number of primary } \\
\text { branches per plant }\end{array}$ & KAK 2, PG071 & PG071, PKC1 & PG071 × GNG1969 & $\begin{array}{c}\text { PG071 } \times \text { PKC1, PG071 } \times \\
\text { GNG1969, GNG1969 } \times \text { HK4 }\end{array}$ & $\begin{array}{c}\text { PG071 } \times \text { PKC1, PG071 } \times \\
\text { GNG1969, GNG1969 } \times \text { HK4 }\end{array}$ \\
\hline 6 & $\begin{array}{c}\text { Number of secondary } \\
\text { branches per plant }\end{array}$ & $\begin{array}{l}\text { PG071, } \\
\text { GNG1969 }\end{array}$ & GNG1969, KAK2 & KAK2 × GNG1969 & $\begin{array}{c}\text { KAK2 } \times \text { GNG1969, KAK2 } \times \text { HK4 } \\
\text { PG071 } \times \text { GNG1969 }\end{array}$ & $\begin{array}{c}\text { KAK2 } \times \text { GNG1969, PG071 } \times \\
\text { GNG1969, KAK } 2 \times \text { HK4 }\end{array}$ \\
\hline 7 & $\begin{array}{l}\text { Number of pods per } \\
\text { plant }\end{array}$ & PG071, KAK 2 & PG071,GNG1969,KAK2 & $\begin{array}{c}\text { PG071 } \times \text { PKC1 } \\
\text { KAK } 2 \times \text { HK } 4\end{array}$ & $\begin{array}{c}\text { PG071 } \times \text { PKC1, PG071 } \times \\
\text { GNG1969, GNG1969 } \times \text { HK4, } \\
\text { JGK-1 } \times \text { HK4, KAK } 2 \times \text { HK4 }\end{array}$ & $\begin{array}{c}\text { PG071 } \times \text { GNG1969, PG071 } \times \\
\text { PKC1, KAK } 2 \times \text { HK4 } \\
\text { GNG1969 } \times \text { HK } 4\end{array}$ \\
\hline 8 & Pod length $(\mathrm{cm})$ & KAK2, PG071 & PKC1,KAK2, PG071 & $\mathrm{PKC} 1 \times \mathrm{HK} 4$ & $\begin{array}{c}\text { JGK1 } \times \text { HK4, PKC1 } 1 \text { HK4, } \\
\text { KAK2 } \times \text { GNG1969, PG071 } \times \\
\text { PKC1, }\end{array}$ & $\mathrm{PKC} 1 \times \mathrm{HK} 4, \mathrm{PG} 071 \times \mathrm{PKC} 1$ \\
\hline 9 & $\begin{array}{l}\text { Number of seeds per } \\
\text { pod }\end{array}$ & GNG1969 & GNG1969 & $\begin{array}{c}\text { PG071 } \times \text { GNG1969, } \\
\text { PG071 } \times \text { PKC1, KAK2 } \times \\
\text { HK4, GNG1969 } \times \text { HK4 }\end{array}$ & $\begin{array}{c}\text { PG071 } \times \text { PKC1, PG071 } \times \\
\text { GNG1969, KAK2 } \times \text { HK4, } \\
\text { GNG1969 } \times \text { HK4 }\end{array}$ & $\begin{array}{c}\text { PG071 } \times \text { PKC1, PG071 } \times \\
\text { GNG1969, KAK2 } \times \text { HK4, } \\
\text { GNG1969 } \times \text { HK4 }\end{array}$ \\
\hline 10 & Grain yield per plant $(\mathrm{g})$ & KAK2, HK4 & KAK2, HK4, PG071 & $\begin{array}{c}\mathrm{PKC} 1 \times \mathrm{HK} 4, \mathrm{KAK} 2 \times \\
\mathrm{HK} 4\end{array}$ & $\begin{array}{l}\mathrm{PKC} 1 \times \mathrm{HK} 4, \mathrm{JGK} 1 \times \mathrm{HK} 4, \\
\mathrm{KAK} 2 \times \mathrm{HK} 4, \mathrm{PKC} 1 \times \mathrm{JGK}-1\end{array}$ & $\begin{array}{c}\mathrm{PKC} 1 \times \mathrm{HK} 4, \mathrm{KAK} 2 \times \\
\text { HK4JGK } 1 \times \mathrm{HK} 4\end{array}$ \\
\hline 11 & $\begin{array}{l}\text { Biological yield per } \\
\text { plant }(\mathrm{g})\end{array}$ & KAK2, HK4 & KAK2, PG071, HK4 & $\begin{array}{c}\text { KAK2 } \times \text { GNG1969, PKC1 } \\
\times \text { HK } 4\end{array}$ & $\begin{array}{c}\text { PKC1 } \times \text { HK4, PKC1 } \times \text { JGK1, } \\
\text { KAK2 } \times \text { GNG1969, JGK-1 } \\
\text { GNG1969 }\end{array}$ & KAK2 $\times$ GNG1969 \\
\hline 12 & 100 seed weight $(\mathrm{g})$ & KAK 2, HK4 & KAK2, HK4 & $\begin{array}{c}\text { KAK } 2 \times \text { HK } 4, \text { PKC } 1 \times \\
\text { HK } 4,\end{array}$ & \begin{tabular}{|} 
JGK1 $\times$ GNG1969, PKC1 $\times$ HK4, \\
GNG1969 $\times$ HK4, KAK2 $\times$ HK4
\end{tabular} & $\mathrm{PKC} 1 \times \mathrm{HK} 4, \mathrm{KAK} 2 \times \mathrm{HK} 4$ \\
\hline 13 & Harvest index (\%) & KAK 2, HK4 & PG071, HK4, KAK2 & $\begin{array}{c}\mathrm{PKC} 1 \times \mathrm{HK} 4, \mathrm{JGK} 1 \times \\
\mathrm{HK} 4\end{array}$ & $\begin{array}{c}\text { PKC1 } \times \text { HK4, JGK1 } 1 \text { HK4, } \\
\text { PKC1 } \times \text { GNG1969, PG071 } \times \\
\text { GNG1969 }\end{array}$ & $\mathrm{PKC} 1 \times \mathrm{HK} 4, \mathrm{JGK} 1 \times \mathrm{HK} 4$ \\
\hline
\end{tabular}




\section{Conclusion}

Thus it is concluded that among six parents taken for diallel analysis, KAK2 was considered as best parent of yield attributes including grain yield per plant followed by parental line PG071 that showed good GCA for eight yield attributes. These promising parents for various traits could be utilized in future breeding programmes in order to create large genetic variability and to isolate true breeding lines with desirable combination of yield attributes. Among crosses, PKC1 $\mathrm{x}$ JGK1 ( $\mathrm{P} \times \mathrm{G})$ and PKC1 x HK4 (G x P) were identified as outstanding crosses for days to flowering and days to maturity, respectively. These crosses can be exploited for isolating early maturity lines. Three crosses viz. PKC1 x HK4 (P x G), KAK2 x HK4 (G x G) and JGK1 x HK4 (P x G) showed significantly high mean performance, good SCA effects and heterosis over mid, better and check parents for grain yield per plant. These crosses were also found superior for other yield components traits. Promising cross combinations based on mean performance, SCA effects and heterosis can be exploited for isolating superior recombinants in the advanced generations. On the basis of cross combinations showing highest heterosis in desired direction and best SCA estimates for most of the characters, it is evident that crosses showing high extent of heterosis have high SCA estimates. This indicated that manifestation of heterosis depends upon SCA estimates. Hence, these crosses could be utilized in future breeding programme.

\section{References}

1. Amadabade J, Arora A, Sahu H. Combining ability analysis for yield contributing characters in Chickpea (Cicer arietinum L.). Electronic journal of Plant Breeding. 2015; 5(4):664-670.

2. Fonseca, S, Patterson F. Hybrid vigour in a seven parent diallel cross in common wheat crop. Crop. Sci. 1968; $1: 85-88$

3. Griffing B. Concept of general and specific combining ability in relation to diallel crossing systems. Australian Journal of Biological Sciences. 1956; 9:463-93.

4. Hays HK, Immer FR, Smith DC. Methods of plant Breeding. Mc Graw Hill Book Co. Inc., New York, 1955, 432.

5. Jadhav AA, Rayate SJ, Mhase LB, Thudi M, Chitikineni, A, Harer PN et al. Marker-trait association study for protein content in chickpea (Cicer arietinum. Journal of genetics. 2016; 94(2):279-286.

6. Moreno MT, Cubero JI. Variation in Cicer arietinum L. Euphytica, 1978; 27(2):465-485.

7. Pandey MP, Tewari SK. Combining ability analysis for yield and its components in chickpea. Egyptian Journal of Genetics and Cytology. 1986; 15(1):69-74.

8. Panse VG, Sukhatme PV. Statistical methods for Agricultural workers. Indian council of Agricultural Research, New Delhi, 1967, 145-152.

9. Van der Maesen LJG. Cicer L. a monograph of the genus, with special reference to the chickpea (Cicer arietinum L.), its ecology and cultivation. Mededlingenlandbouwhoge School (Communication Agricultural University) Wageningen. 1972; 72-10:342. 